\title{
Ethnografische Qualifikationsarbeiten im Setting Schule - das Spannungsfeld zwischen Forschungsethik, Datenschutz und Forschungspraxis
}

\author{
Veronika Knoche
}

Eingegangen: 3. Juli 2020 / Angenommen: 18. Mai 2021 / Online publiziert: 21. Oktober 2021

(C) Der/die Autor(en) 2021

Zusammenfassung Das hier vorgestellte Dissertationsprojekt beschäftigt sich mit der Frage, wie Schulsozialarbeiter*innen ihr professionelles Handeln im beruflichen Alltag reflektieren. In der Vorbereitung der ethnografischen Feldphase zeigten sich forschungsethische, datenschutzrechtliche und forschungspraktische Fragestellungen. Der Kontext Schule als sensibles Forschungsfeld und die Rahmenbedingungen einer Qualifikationsarbeit bildeten die Grundlage der Fragestellungen im Hinblick auf die konkrete Umsetzung guter wissenschaftlicher Praxis. Die Beschreibung dieses Spannungsfeldes und der eingeschlagenen Lösungswege soll als Anregung dienen, um den Austausch über qualitative Sozialforschung und deren Rahmenbedingungen voranzutreiben.

Schlüsselwörter Forschungsethik · Forschungspraxis · Datenschutz · Ethnografie · Schulsozialarbeit

\section{Ethnographic dissertations in school settings-the area of tension between research ethics, data protection and research practice}

\begin{abstract}
The presented research project seeks to find out how school social workers reflect on their professional actions in everyday professional life. During preparations for the ethnographic field phase, dilemmas between research ethics, data privacy protection, and research practice emerged. Research in the school setting and the frame of dissertation work formed the basis for the implementation of good scientific research. The description of this area of tension and the proposed solutions could provide a stimulus for the scientific community to deepen the exchange on qualitative social research and its framework conditions.
\end{abstract}

Veronika Knoche $(\square)$

Lehrstuhl für Sozialpädagogik, Otto-Friedrich-Universität Bamberg, Bamberg, Deutschland

E-Mail: Veronika.knoche@uni-bamberg.de 
Keywords Research ethics · Research practice - Data privacy protection · Ethnography $\cdot$ School social work

\section{Hintergrund}

Die Schulsozialarbeit gilt als eines der am intensivsten beforschten Arbeitsfelder der Kinder- und Jugendhilfe (Speck und Olk 2010a). Dennoch fehlen differenzierte Erkenntnisse zu grundlegenden Themen wie Verbreitung, Entwicklung, erreichte Wirkungen, aber auch zu methodischem Handeln und professionellen Standards in der Schulsozialarbeit (Speck und Olk 2010b; Speck 2014, S. 166). Berufliches Selbstverständnis und Handlungsorientierungen von Schulsozialarbeiter*innen wurden bisher kaum erforscht (Haase 2017, S. 340; Speck und Olk 2010b, S. 7). Das Forschungsprojekt bringt deshalb in Erfahrung, wie professionelles Handeln im beruflichen Alltag reflektiert wird, welche Facetten der Ausgestaltung von Professionalität in der Praxis in Erscheinung treten und an welche bisherigen professionstheoretischen Überlegungen diese anschlussfähig sind. ${ }^{1}$ Bezogen auf den Professionalisierungsdiskurs in der Sozialen Arbeit (Becker-Lenz et al. 2013) sind Bezüge zu den Überlegungen zur Reflexiven Professionalität (Dewe 2009), dem Handlungskompetenzmodell von Heiner (2016) oder Staub-Bernasconis Kritischer Professionalität (2018) denkbar. Da Reflexionsfähigkeit als wichtige Kompetenz professionellen Handelns betrachtet werden kann (Ebert 2012), bieten die in der Forschungsarbeit herausgearbeiteten Reflexionsstrategien Hinweise auf die Gestaltung notwendiger Rahmenbedingungen professioneller Schulsozialarbeit.

\section{Forschungsdesign und damit verbundene Herausforderungen}

Um diese Fragestellungen zu beantworten, wurde ein ethnografischer Zugang und der Forschungsstil der Grounded Theory nach Strauss und Corbin (1996) gewählt. Die Schulsozialarbeiter*innen, welche sich nach einem Aufruf über den E-MailVerteiler eines Dachverbands der Kinder- und Jugendhilfe freiwillig für das Forschungsprojekt meldeten, wurden an mehreren Tagen ethnografisch in teilnehmender Beobachtung im beruflichen Alltag begleitet. Bereits die Vorbereitungen führten zu besonders spannungsreichen Aspekten. Diese Herausforderungen ethnografischer Forschung im Setting Schule und die eingeschlagenen Lösungswege werden hier kurz skizziert.

Gegenstand der Forschung waren die Schulsozialarbeiter*innen als Tätige einer Einrichtung der Kinder- und Jugendhilfe, weshalb die Erhebung formal in erster Linie durch die jeweiligen freien und öffentlichen Träger der Kinder- und Jugendhilfe genehmigt werden musste. Kooperationsvereinbarungen zwischen Träger und Schule regeln den Einsatzort der Schulsozialarbeiter*innen, sodass zusätzlich an jeder Schule die Erlaubnis der Schulleitung erforderlich war. Angesichts der gesellschaft-

\footnotetext{
1 Für einen Überblick über relevante Positionen innerhalb des Professionsdiskurses siehe Pfadenhauer und Sander (2010).
} 
lichen Diskussion und fortwährenden Alltagspräsenz der Datenschutz-Grundverordnung (DSGVO; Europäisches Parlament und Europäischer Rat 27.04.2016) erschien dessen Berücksichtigung für die Feldphase offensichtlich, sodass zunächst eine Einwilligungserklärung für die Verarbeitung personenbezogener Daten der Schulsozialarbeiter*innen erstellt wurde. In der Ausarbeitung dieser mithilfe des Datenschutzbeauftragten der Universität Bamberg stellte sich die Frage nach weiteren Beteiligten im Feld, den zum Großteil minderjährigen Schüler*innen, deren Sorgeberechtigten sowie den Lehrer*innen. Ethnografische Forschung in ihrer wenig institutionalisierten Form geht mit vielen kurzen, anonymen Feldkontakten (z. B. auf dem Pausenhof) und informellen Gesprächen einher. Im Forschungsprojekt wurde daher zwischen unterschiedlich stark von der Forschung betroffenen Gruppen differenziert. Von Unger et al. weisen in diesem Zusammenhang darauf hin, dass der Grundsatz der informierten Einwilligung in der angloamerikanischen Literatur ausführlich reflektiert wurde (2016; Bell 2014; Fluehr-Lobban 2013; Shannon 2007). Dort stellte sich die Frage, ,ob dieser Grundsatz, der für medizinische und quantitative Forschung entwickelt wurde, in der ethnografischen Forschung, wo Zugang zum Feld, Teilnahme und Kommunikation anders organisiert sind, überhaupt angemessen ist" (von Unger et al. 2016). Die zunächst rein forschungspraktisch erschienene Hürde, die informierte Einwilligung in einem offenen Beobachtungskontext umzusetzen, verwies also auf eine forschungsethische Komponente. Für Fragen der Forschungsethik sind Ethikkommissionen die richtigen Ansprechpartner. Die Möglichkeit der Diskussion forschungsethischer Aspekte mittels Begutachtung des Forschungsprojekts durch die Ethikkommission der DGSA ist derzeit jedoch für Qualifikationsarbeiten nicht vorgesehen, sondern liegt in der Verantwortung der Betreuer*innen (ForschungsethikKommission der DGSA). Für die Sicherstellung guter wissenschaftlicher Praxis im Sinne eines verantwortungsvollen Umgangs mit der verfassungsrechtlich gesicherten Forschungsfreiheit ist die ausführliche Reflexion der forschungsethischen Aspekte jedoch unabdingbar (Deutsche Forschungsgemeinschaft 2019, S. 16).

\section{Forschungspraktische Lösungsansätze}

Im vorgestellten Forschungsprojekt wurden die Forschungsziele und -fragen unter Berücksichtigung der Kriterien guter wissenschaftlicher Praxis zur „Richtschnur“ für die forschungspraktische Umsetzung des Forschungsvorhabens. Anhand der Fragestellung „Wie reflektieren Schulsozialarbeiter*innen ihr professionelles Handeln im beruflichen Alltag?" wurde zwischen direkt und indirekt beforschten Personengruppen unterschieden. Die Schulsozialarbeiter*innen als direkt beforschte Personen wurden gemäß des Forschungsethikkodex der DGSA ${ }^{2}$ (Deutsche Gesellschaft für Soziale Arbeit 2020) ausführlich über das Forschungsvorhaben informiert, bevor sie schriftlich in die Teilnahme einwilligten. Das Prinzip der minimalen Risiken (ebd.) erfordert Sensibilität für mögliche Risiken und Belastungen der Forschungs-

\footnotetext{
2 Der Forschungsethikkodex der DGSA wurde per Online-Wahl im Juli 2020 verabschiedet. Die Entscheidung des Vorstands der DGSA, diese Entscheidung nicht auf eine Mitgliederversammlung in Präsenz zu verschieben, unterstreicht den dringenden Bedarf einer soliden Arbeitsgrundlage in diesem Bereich.
} 
teilnehmer*innen und deren Minimierung. Zum Schutz der Personen wurden daher die Namen und Einsatzorte der direkt beforschten Personen ausschließlich anonymisiert in die Beobachtungsprotokolle aufgenommen, sodass keine Rückschlüsse auf Personen möglich waren. Die indirekt beforschten Personen stellten eine große, heterogene Gruppe dar, welche aus Schüler*innen, Sorgeberechtigten, Lehrer*innen und weiteren Beteiligten bestand. Unter ihnen wurden die Erwachsenen über den an der jeweiligen Schule üblichen Informationsweg (z. B. Elternbrief, Newsletter) über das Forschungsprojekt informiert, während Kinder und Jugendliche gemeinsam von der Forscherin und den beforschten Schulsozialarbeiter*innen im Gespräch informiert wurden. Alle Personen wurden sowohl über die Anonymität ihrer Äußerungen als auch Möglichkeit der Nicht-Teilnahme aufgeklärt und konnten frei entscheiden, ob die Forscherin bei Gesprächen anwesend sein durfte. Auch bei spontanen Gesprächen der Schulsozialarbeiter*innen mit minderjährigen Kindern, der häufigsten Form der Beratung in der Schulsozialarbeit, konnten die Kinder selbst wählen, ob die Forscherin anwesend sein durfte. Von Seiten der Forscherin wurde Sorge getragen, potenzielle Risiken sozialer Zwänge, bspw. durch die beforschten Schulsozialarbeiter*innen, zu minimieren. Dies erfolgte vor allem durch Aufklärung der beforschten Personen, dass die Nicht-Teilnahme einzelner Kinder keinen negativen Einfluss auf die Forschung habe. Absolute Sicherheit über die tatsächlich von Schüler*innen empfundene Entscheidungsfreiheit oder im Verborgenen stattgefundene negative Konsequenzen bspw. durch Eltern, Peers oder eigene Ansprüche kann im Normalfall nicht erreicht werden. Einige Ablehnungen der Teilnahme der Forscherin an Gesprächen zeigen jedoch, dass die Aufklärung darüber, dass bei NichtTeilnahme keine negativen Konsequenzen drohen, zur Minimierung des Risikos des sozialen Zwangs beiträgt. In diesen Fällen wurden die Betroffenen auch anonym nicht in den Beobachtungsprotokollen erwähnt. Aufgrund der geringen Risiken konnte davon ausgegangen werden, dass auch Kinder im jungen Grundschulalter diese Entscheidung treffen konnten. Von den indirekt beforschten Personen wurden keine personenbezogenen Daten aufgenommen, sondern ausschließlich Inhalte und Interaktionen während der Gespräche mit den Schulsozialarbeiter*innen verarbeitet.

\section{Resümee}

Intensive Auseinandersetzung mit und sorgfältige Abwägung von forschungsethischen, datenschutzrechtlichen und forschungspraktischen Aspekten stellen wesentliche Aufgaben in der Vorbereitung der Feldphase ethnografischer Forschungsvorhaben dar. Jede Forscherin/jeder Forscher ist gefordert, diesen Anforderungen in eigener Verantwortlichkeit (und bei Qualifikationsarbeiten in Absprache mit den Betreuer*innen der Arbeit) gerecht zu werden (Forschungsethik-Kommission der Deutschen Gesellschaft für Soziale Arbeit (DGSA)). Ethnografische Forschungsprojekte zur Schulsozialarbeit sind bisher nur mit hoch individualisierten forschungspraktischen Lösungen durchführbar, obwohl diese einen wichtigen Beitrag für die noch lückenhafte Grundlagenforschung in der Schulsozialarbeit leisten könnten. Um Forschungsprojekte zum Thema Schulsozialarbeit zu fördern und so perspektivisch einen Teil der bisherigen Forschungslücken schließen zu können, wäre die Entwick- 
lung von Richtlinien für die Gestaltung ethnografischer Forschung im Setting Schule wünschenswert.

Funding Open Access funding enabled and organized by Projekt DEAL.

Open Access Dieser Artikel wird unter der Creative Commons Namensnennung 4.0 International Lizenz veröffentlicht, welche die Nutzung, Vervielfältigung, Bearbeitung, Verbreitung und Wiedergabe in jeglichem Medium und Format erlaubt, sofern Sie den/die ursprünglichen Autor(en) und die Quelle ordnungsgemäß nennen, einen Link zur Creative Commons Lizenz beifügen und angeben, ob Änderungen vorgenommen wurden.

Die in diesem Artikel enthaltenen Bilder und sonstiges Drittmaterial unterliegen ebenfalls der genannten Creative Commons Lizenz, sofern sich aus der Abbildungslegende nichts anderes ergibt. Sofern das betreffende Material nicht unter der genannten Creative Commons Lizenz steht und die betreffende Handlung nicht nach gesetzlichen Vorschriften erlaubt ist, ist für die oben aufgeführten Weiterverwendungen des Materials die Einwilligung des jeweiligen Rechteinhabers einzuholen.

Weitere Details zur Lizenz entnehmen Sie bitte der Lizenzinformation auf http://creativecommons.org/ licenses/by/4.0/deed.de.

\section{Literatur}

Becker-Lenz, R., Busse, S., Ehlert, G., \& Müller-Hermann, S. (Hrsg.). (2013). Professionalität in der Sozialen Arbeit. Standpunkte, Kontroversen, Perspektiven. Wiesbaden: Springer VS.

Bell, K. (2014). Resisting Commensurability: Against Informed Consent as an Anthropological Virtue. American Anthropologist, 116(3), 511-522. https://doi.org/10.1111/aman.12122.

Deutsche Forschungsgemeinschaft (2019). Leitlinien zur Sicherung guter wissenschaftlicher Praxis. Kodex. Unter Mitarbeit von: Wissenschaftliche Integrität Gruppe Chancengleichheit. Bonn: Deutsche Forschungsgemeinschaft.

Deutsche Gesellschaft für Soziale Arbeit (DGSA) (2020). Forschungsethische Prinzipien und wissenschaftliche Standards für Forschung der Sozialen Arbeit Forschungsethikkodex der DGSA. Verabschiedet durch die Mitglieder im Juli 2020

Dewe, B. (2009). Reflexive Professionalität. In A. Riegler (Hrsg.), Soziale Arbeit zwischen Profession und Wissenschaft. Vermittlungsmöglichkeiten in der Fachhochschulausbildung (S. 47-63). Wiesbaden: VS.

Ebert, J. (2012). Reflexion als Schlüsselkategorie professionellen Handelns in der Sozialen Arbeit (2. Aufl.). Hildesheimer Schriften zur Sozialpädagogik und Sozialarbeit, Bd. 16. Hildesheim, Zürich, New York: Georg Olms Verlag.

Europäisches Parlament und Europäischer Rat (2016). Verordnung (EU) 2016/679 des Europäischen Parlaments und des Rates vom 27. April 2016 zum Schutz natürlicher Personen bei der Verarbeitung personenbezogener Daten, zum freien Datenverkehr und zur Aufhebung der Richtlinie 95/46/EG (Datenschutz-Grundverordnung). EU-DSGVO. In: Amtsblatt der Europäischen Union (L 119), S. 1-88. Online verfügbar unter https://dsgvo-gesetz.de/.

Fluehr-Lobban, C. (2013). Ethics and anthropology. Ideas and practice. Lanham: AltaMira Press. Online verfügbar unter. http://site.ebrary.com/lib/alltitles/docDetail.action?docID=10780911.

Forschungsethik-Kommission der Deutschen Gesellschaft für Soziale Arbeit (DGSA) Forschungsethik-Kommission: Begutachtung von Forschungsvorhaben. Hg. v. Deutsche Gesellschaft für Soziale Arbeit (DGSA. https://www.dgsa.de/ueber-uns/forschungsethik-kommission/. Zugegriffen: 13.10.2021.

Haase, K. (2017). Das berufliche Selbstverständnis in der Schulsozialarbeit. Zu Gast in einem fremden Haus? Soziale Arbeit: Zeitschrift Für soziale und Sozialverwandte Gebiete, 66(9), 339-345.

Heiner, M. (2016). Kompetent handeln in der Sozialen Arbeit (2. Aufl.). Handlungskompetenzen in der Sozialen Arbeit, Bd. 1. München, Basel: Ernst Reinhardt Verlag.

Pfadenhauer, M., \& Sander, T. (2010). Professionssoziologie. In: Georg Kneer und Markus Schroer (Hg.): Handbuch Spezielle Soziologien. Wiesbaden: VS/ GWV, S. 361-378. 
Shannon, J. (2007). Informed consent: documenting the intersection of bureaucratic regulation and ethnographic practice. Political and Legal Anthropology Review, 30(2), 229-248.

Speck, K. (2014). Schulsozialarbeit. Eine Einführung (3. Aufl.). München: Ernst Reinhardt Verlag.

Speck, K., \& Olk, T. (Hrsg.). (2010a). Forschung zur Schulsozialarbeit. Stand und Perspektiven. Weinheim, München: Juventa Verlag. https:/www.pedocs.de/volltexte/2013/7094/pdf/Speck_Olk_Sltimpel_ Auf_dem_Weg_zu_multiprofessionellen_Organisationen.pdf. Zugegriffen: 13.10.2021.

Speck, K., \& Olk, T. (2010b). Zur Forschung in der Schulsozialarbeit. In K. Speck \& T. Olk (Hrsg.), Forschung zur Schulsozialarbeit. Stand und Perspektiven (S. 7-8). Weinheim, München: Juventa.

Staub-Bernasconi, S. (2018). Soziale Arbeit als Handlungswissenschaft. Auf dem Weg zu kritischer Professionalität. Opladen, Toronto: Barbara Budrich.

Strauss, A.L., \& Corbin, J. M. (1996). Grounded Theory. Grundlagen qualitativer Sozialforschung. Weinheim: Beltz.

von Unger, H., Dilger, H., \& Schönhuth, M. (2016). Ethikbegutachtung in der sozial- und kulturwissenschaftlichen Forschung? Ein Debattenbeitrag aus soziologischer und ethnologischer Sicht. Forum Qualitative Sozialforschung, 17(3). https://www.ratswd.de/working-paper/259. Zugegriffen: 13.10.2021. 